\title{
Article
}

\section{Knee joint coordination during single-leg landing in different directions}

Sinsurin, Komsak, Vachalathiti, Roongtiwa, Srisangboriboon, Sarun and Richards, James

Available at http://clok.uclan.ac.uk/24497/

Sinsurin, Komsak, Vachalathiti, Roongtiwa, Srisangboriboon, Sarun and Richards, James ORCID: 0000-0002-4004-3115 (2020) Knee joint coordination during single-leg landing in different directions. Sports Biomechanics, 19 (5). pp. 652-664. ISSN 1476-3141

It is advisable to refer to the publisher's version if you intend to cite from the work. http://dx.doi.org/10.1080/14763141.2018.1510024

For more information about UCLan's research in this area go to http://www.uclan.ac.uk/researchgroups/ and search for <name of research Group>.

For information about Research generally at UCLan please go to http://www.uclan.ac.uk/research/

All outputs in CLoK are protected by Intellectual Property Rights law, including Copyright law. Copyright, IPR and Moral Rights for the works on this site are retained by the individual authors and/or other copyright owners. Terms and conditions for use of this material are defined in the policies page. 


\title{
Title Page
}

\section{Knee Joint Coordination during Single-leg Landing in Different Directions}

\author{
Komsak Sinsurin $^{\mathrm{a}}$, Roongtiwa Vachalathiti ${ }^{\mathrm{a}}$, Sarun Srisangboriboon ${ }^{\mathrm{a}}$, Jim Richards ${ }^{\mathrm{b}}$ \\ ${ }^{a}$ Biomechanics and Sports Research unit, Faculty of Physical Therapy, Mahidol University, \\ Thailand \\ ${ }^{\mathrm{b}}$ Allied Health Research unit, University of Central Lancashire, UK
}

\section{Corresponding Author:}

Associate Professor Roongtiwa Vachalathiti, PT, PhD

Faculty of Physical Therapy, Mahidol University, 999 Phuttamonthon 4 Road, Salaya, Thailand.

E-mail: roongtiwa.vac@mahidol.ac.th

Tel: +6624415450 \#20401 Fax: +6624415454

Word count: 2845 words

\section{Acknowledgements}

The authors would like to thank all the athletes for participation in this study.

\section{Funding}

This research project is supported by Mahidol University. 


\section{Abstract}

2 Knee joint coordination during jump landing in different directions is an important consideration

3 for injury prevention. The aim of the current study was to investigate knee and hip kinematics on

4 the non-dominant and dominant limbs during landing. Nineteen female volleyball athletes

5 performed single-leg jump landing tests in four directions; forward $\left(0^{\circ}\right)$, diagonal $\left(30^{\circ}\right.$ and $\left.60^{\circ}\right)$,

6 and lateral $\left(90^{\circ}\right)$ directions. Kinematic and ground reaction force (GRF) data were collected using

7 a 10-camera Vicon system and an AMTI force plate. Knee and hip joint angles, and knee angular

8 velocities were calculated using a lower extremity model in Visual3D. A two factor repeated

9 measures ANOVA was performed to explore limb dominance and jump direction. Significant

10 differences were seen between the jump directions for; angular velocity at initial contact ( $\mathrm{p}<$

$110.001)$, angular velocity at peak VGRF $(\mathrm{p}<0.001)$, and knee flexion excursion $(\mathrm{p}=0.016)$. Knee

12 coordination was observed to be poorer in the early phase of velocity-angle plot during landing in

13 lateral direction compared to forward and diagonal directions. The non-dominant limb seemed to

14 have better coordination than the dominant limb during multi-direction jump landing. Therefore,

15 dominant limbs appear to be at a higher injury risk than non-dominant limbs.

17 Keywords: knee stability, knee angular velocity, single-leg landing, volleyball athletes 


\section{Introduction}

Landing from jumps can induce moderate strain forces to the structures of the knee due to

27 the complex and aggressive nature of such tasks (Boden, Dean, Feagin, \& Garrentt, 2000;

28 Kirkendall \& Garrett, 2000). These can lead to knee injuries such as anterior cruciate ligament

29 (ACL) injury, which have been frequently reported during landing (Hootman, Dick, \& Aqel, 2007).

30 A 'soft-style landing' with greater knee and hip flexion, has been shown to reduce ground reaction

31 forces (Devita \& Skelly, 1992), which in turn has been shown to decrease loading of the ACL (Yu,

32 Lin, \& Garrett, 2006).

33 Joint coordination may be described as the ability of the muscles to control a joint during

34 dynamic tasks such as landing. Measures of joint coordination may provide a greater insight into

35 the motor control by the central nervous system (Scholz, 1990). Coordination may also be

36 described as the ability to reduce joint loading during movement through improved dynamic

37 stability (William, Chmielewski, Rudolph, Buchanan, \& Snyder-Mackler, 2001). William et al.

38 proposed that dynamic knee stability depends on articular geometry, soft tissue restraints, and joint

39 loading from both weight bearing and muscle forces. Therefore, any increases in knee stability

40 during landing may be as a result of improved coordination, and any fluctuation of movement

41 variability may represent poor coordination. However, in contrast, previous study reported that

42 atypically increases or decreases in variability may be the cause of injury (Robertson, Caldwell,

43 Hamill, Kamen, Whittlesey, 2014). This supported Kurz and Stergious (2004), who suggested that

44 abnormal movement patterns during movement perturbations could be observed in an unhealthy

45 system, indicating an inability to adapt or control movement in multiple degrees of freedom.

Previously angle-angle plots and velocity-angle plots (phase plane plot) have been used to

47 measure lower limb and joint coordination (Bartlett \& Bussey, 2012). The use of angle-angle

48 diagram was first proposed by Grieve (1986) as a simple technique for analysing the interaction of 
49 the angle data from two joints. These plots allow a representation of movement coordination of 50 two joints and how they 'co-vary' which can be used to compare coordination patterns between

51 conditions, and to focus on how the joint changes with respect to an adjacent joint. Phase plane

52 plots offer a representation of the interaction between joint velocity and angle. These may be used

53 to identify changes in joint control and coordination characteristics (Robertson, Caldwell, Hamill,

54 Kamen, Whittlesey, 2014). Excessive variation of movement pattern or poor coordination has been

55 associated with instabilities which are the result of neuromuscular impairment (Clark and Phillips,

56 1993), such as in gait of people with Parkinson disease. Heidersciet et al. (2002) demonstrated that

57 the coordination variability of the thigh/leg movement was different between individuals with and

58 without patellofemoral pain, with reduced variability representation movement compensation due

59 to pain.

60

Various directions of landing can be observed in different sporting activities. Previous

61 studies have shown differences in lower limb biomechanics during multi-directional landing

62 (Sinsurin, Srisangboriboon, \& Vachalathiti, 2017; Sinsurin et al., 2013; Sinsurin, Vachalathiti,

63 Jalayondeja, \& Limroongreungrat, 2016). However, assessment of differences in knee and hip

64 coordination during jump landing in different directions has not been reported to date. This should

65 provide a greater understanding of the knee coordination when performing different directions of

66 jump which could highlight important considerations for injury prevention. Therefore, the aim of

67 the current study was to investigate knee coordination during landing in various directions, and to

68 compare landing on the non-dominant knees and dominant knees. We hypothesised that differences

69 in knee and hip kinematics exist between jump-landing direction and between dominant and non-

70 dominant limbs. 


\section{Methods}

\section{$74 \quad$ Participants}

Twenty-one female volleyball athletes were recruited. All had participated in the university

76 team and had no report of musculoskeletal problems on either leg in the three months prior to

77 testing. Exclusion criteria included any serious injury or surgery to the lower extremities, such as

78 ankle sprain, ACL injury, fracture, or patellar dislocation. Testing procedures were explained to all

79 participants. Each participant read and signed an informed consent form, which was approved by

80 the Committee on Human Rights Related to Human Experimentation of Mahidol University (COA.

81 No. 2013/045.1705).

82 A power calculation identified that 21 participants were required to provide a statistical 83 power of $85 \%$ and an effect size of 0.3 calculated from pilot data of 5 volleyball athletes. However,

84 data was incomplete for 2 participants, therefore data from only 19 participants was reported. The 85 athletes' average age and experience were $19.7 \pm 1.4$ years and $9.6 \pm 2.0$ years, respectively, and

86 all participants were right-leg dominant. The dominant limb was defined by the single-leg hop for

87 distance protocol, which determined the longest hop distance for the dominant side (van der Harst,

88 Gokeler, \& Hof, 2007). In addition, height, body weight, leg length, knee width, and ankle width 89 were recorded.

\section{$91 \quad$ Jump-Landing Tests}

92 Multi-directional jump landing tests were collected in a Motion Analysis Laboratory.

93 Kinematic data were recorded using a 10 camera Vicon ${ }^{\mathrm{TM}}$ Nexus system (Oxford Metrics, Oxford,

$94 \mathrm{UK}$ ) at $100 \mathrm{~Hz}$, and force data were collected using an AMTI force plate (Advanced Mechanical

95 Technology, Massachusetts, USA) at 1,000 Hz. The force plate was used to define the events of an 96 initial contact and peak vertical ground reaction force (VGRF). Sixteen reflective markers were 
97 placed bilaterally on the lower-limb bony prominences of participants including; anterior superior

98 iliac spines, posterior superior iliac spines, thighs, lateral condyles of the femurs, shanks, lateral

99 malleoli, heels, and the head of the $2^{\text {nd }}$ metatarsal bones. A 30 -cm-height wooden platform was

100 placed $70 \mathrm{~cm}$ from the centre of the force plate.

101 Tillman et al. (2004) reported that unilateral landing was 50\% approximately in volleyball.

102 This supports the use of unilateral jump-landing test as an appropriate assessment of the risk of

103 lower extremity landing injuries (Sinsurin et al., 2013; Sinsurin et al., 2017; Tamura, Akasaka,

104 Otsudo, Schiozawa, Toda, \& Yamada, 2017). Therefore, this study examined jump-landing test

105 with one leg. The participants stood on the platform on the leg to be tested and flexed the other

106 knee approximately $90^{\circ}$ with a neutral hip rotation. To eliminate variability in jumping mechanics

107 due to arm-swing, the participants were asked to place both hands on their waist. Each participant

108 was instructed to carefully jump off the wooden platform without an upward jump action in order

109 to standardised the jump height between jump-landing tests in four directions. Four randomised

110 directions were used; forward $\left(0^{\circ}\right)$, diagonal $\left(30^{\circ}\right.$ and $\left.60^{\circ}\right)$, and lateral $\left(90^{\circ}\right)$ (Figure 1). These have

111 been previously used by Sinsurin et al. (2013), who showed that jump-landing direction influenced

112 lower extremity biomechanics. The participants jumped and landed with the tested leg while always

113 facing and looking forward during the jump-landing tests. A successful trial was collected if the

114 participant was able to land on the centre of the force plate, maintain unilateral balance, and

115 maintain their hands on their waist. Unsuccessful trials were excluded, and the jump-landing test

116 was repeated. The participants were allowed up to five practice jumps landing in each direction

117 before the recorded trials. Participants were allowed to rest for five minutes between test directions

118 and for at least thirty seconds between individual jumping trials. 


\section{Data Acquisition and Statistical Analysis}

The kinematic and force plate data were filtered using a fourth-order zero-lag Butterworth

123 digital filter at cut-off frequencies of $6 \mathrm{~Hz}$ and $40 \mathrm{~Hz}$, respectively. The cut-off frequency was

124 determined by the residual analysis technique (Winter, 2005). A three-dimensional model was

125 constructed using Visual3D version 6 (C-Motion Inc., USA). The average of three successful trials

126 in each direction for each limb was analysed. The landing phase was identified from the initial

127 contact to $300 \mathrm{~ms}$ after initial contact. Knee and hip joint kinematics were calculated based on the

128 cardan sequence of XYZ, equivalent to the joint coordinate system proposed by Grood and Suntay

129 (1983). Knee-hip angle-angle plots, knee velocity-angle plots, knee flexion excursion, and knee

130 angular velocity at initial contact and at peak VGRF were reported. Knee flexion excursion was

131 calculated from an angular displacement from an initial contact to peak knee flexion during landing 132 phase.

133 Statistical analysis was performed using SPSS version 17. Repeated-measure ANOVA (2

$134 \times 4$, side $\times$ jump-landing direction) were used to determine the effect of limb jump-landing

135 direction and knee side. In addition, post hoc pairwise comparisons were performed to compare

136 the landing directions. The statistical significance was set at an alpha level of 0.05 .

\section{Results}

139 No significant interactions were seen between limb and direction of landing and no

140 significant differences were seen between the dominant and non-dominant limbs. However, the

141 direction of jump landing significantly affected knee angular velocity at initial contact with the

142 greatest velocity seen during the 0 degree jump and the lowest at 90 degrees $(\mathrm{F}(1.388,24.986)=$

143 64.447, $\mathrm{p}<0.001)$. Conversely the greatest knee angular velocity at peak VGRF was seen during

144 the 90 degrees jump and the lowest at 0 and 30 degrees $(F(2.007,36.127)=16.583, p<0.001)$. 
145 Whereas knee flexion excursion showed the lowest value during the 90 degrees jump $(\mathrm{F}(3,54)=$

$1463.750, p=0.016)$. Further analysis of the patterns of knee flexion angle, knee angular velocity, hip-

147 knee angle-angle plot, and knee velocity-angle plots showed similar patterns for the non-dominant

148 and dominant limbs. However, non-dominant and dominant limbs revealed different movement

149 strategies between the different jump directions, Figures 2-6.

150

151 Discussion and Implications

152 The purpose of this study was to examine how knee joint coordination on the non-dominant

153 and dominant limbs respond during landing in various directions. Sagittal plane knee kinematics

154 included knee angular velocity at initial contact and at peak VGRF, and knee flexion excursion.

155 Moreover, differences in coordination during landing of the hip-knee angle-angle and knee 156 velocity-angle plots were explored.

157 Greater flexion of the knee and hip joints has been shown to help to reduce GRF during 158 landing (Onate, Guskiewicz, \& Sullivan, 2001; Cronin, Bressel, \& Fkinn, 2008). A key finding of 159 this study was that that jump-landing direction significantly influenced flexion excursion and 160 angular velocity of the knee. The difference of knee flexion excursion between directions was 161 small, albeit significant, with less excursion of knee flexion noted in lateral direction for both limbs 162 compared to other directions (Figure 2). However, a maximum difference of 2.4 degrees between 163 landing directions could not be considered as clinical important (Table 1).

164 At initial contact, significant differences were seen between landing directions with a trend 165 of decreasing knee angular velocity observed from forward, diagonal, and lateral direction, 166 respectively (Table 1). In addition, on average the knee angular velocity on the non-dominant limb 167 was lower than the dominant, although no significant differences were seen between limbs. 168 Previous studies (Sinsurin et al., 2013; Sinsurin et al., 2017) exhibited that lateral jump landing 
169 needed higher knee flexion at initial contact than forward and diagonal directions. They suggested

170 that lateral jump landing has the higher risk of knee injury compared to forward and diagonal

171 directions. Indicating that athletes preferred a strategy of increased knee flexion at initial contact

172 to prevent knee injury. Therefore, the increased knee flexion and decreased knee angular velocity

173 at initial contact would be the preferred strategy of normal knee control responding jump landing

174 in forward, $30^{\circ}$ diagonal, $60^{\circ}$ diagonal, and lateral directions, respectively.

175 Previously, it has been reported that an increase of lower limb flexion during a soft-style

176 landing helps to control body downward motion more effectively (Laughlin et al., 2011; Favre,

177 Clancy, Dowling, \& Andriacchi, 2016). Our data shows that, after foot contact, knee flexion

178 progressively increased (Figure 2) while angular velocity showed a trend of decrease in all

179 directions except with lateral direction (Figure 3). At peak VGRF, a significant greater knee angular

180 velocity of both limbs was noted in lateral direction compared to other directions (Table 1). This

181 finding would indicate that the better control of the knee during landing was noted in forward

182 direction followed by the diagonal and lateral directions. Even though athletes have the strategy to

183 prevent knee injury with increased flexion angle and decreased angular velocity at initial contact,

184 greater angular velocity during landing phase was observed in lateral jump landing (Figure 3 ). This

185 could be the result from poor control of eccentric contraction of knee extensor muscles in lateral

186 jump landing compared to other directions (Figure 3). This was the phenomenon of knee control

187 in healthy volleyball athletes, and it could be that the risk of knee injury might be higher in athletes

188 who have asymptomatic musculoskeletal problems, especially when landing in lateral direction.

189 Hip-knee angle-angle diagrams offer a representation of the movement coordination which

190 was compared qualitatively between conditions (Bartlett \& Bussey, 2012). In addition, the

191 smoothness of movement may also be observed during movements in such angle-angle plots

192 (Richards, 2008). The current study focused on how the knee flexion changed with a change in the 
193 hip flexion and how these 'co-vary' during landing, Figure 4. A linear relationship was observed

194 as 'in-phase' coordination. Increased knee flexion was observed while hip flexion increased during

195 landing for all jump-landing directions and sides. Comparing between directions, all plots showed

196 a smooth trend of increase for both sides. However, hip and knee muscular coordination responded

197 differently in jump-landing direction constraint, with the coordinative response in lateral direction

198 appearing to be different from the other directions. In particular, less hip-knee flexion-flexion angle

199 was noted during the lateral jump landing (Figure 4), which would indicate a greater stiffness of

200 the lower limb through the landing phase. Previous studies have reported an increased risk of lower

201 limb injuries with a higher joint stiffness, indicating poorer energy dissipation during landing

202 (Zhang, Bates, \& Dufek, 2000). Moreover, in lateral direction, displacement of knee flexion was

203 greater than hip flexion compared to other directions for both limbs in the late phase of landing.

204 This might indicate that athletes need to keep lower center of mass position to maintain body

205 stability in lateral direction compared to other directions.

206 The knee coordination during landing phase was reported in terms of knee velocity-angle

207 or phase plane plot. Comparing patterns between directions in Figure 5, the knee velocity-angle

208 plot in the lateral direction was notably different from other directions. In lateral direction, knee

209 angular velocity progressively increased from initial contact to $35^{\circ}$ knee flexion during landing,

210 whereas forward and diagonal demonstrated a progressive decrease of knee angular velocity

211 indicating that knee extensor muscle worked eccentrically with difficulty to control dynamic knee

212 flexion during lateral jump landing. With greater the control difficulty there is a higher risk of knee

213 injury, which would be exacerbated if athletes landed awkwardly or had a poor balance during

214 landing in lateral direction. Comparing patterns of knee velocity-angle plot between the non-

215 dominant and dominant limbs, Figure 6, knee angular velocity-angle plots exhibited a similar 216 pattern in each of the jump directions. Although a higher angular velocity was observed in the 
217 dominant compared to the non-dominant limbs for all directions of jump landing. Previous studies

218 suggested that non-dominant limbs get used to weight-bearing and therefore have the less risk of

219 knee injury than dominant limbs (Ross, Guskiewicz, Prentice, Schneider, \& Yu, 2004). In addition,

220 the findings from this current study are supported by Sinsurin et al., (2017) who reported that non-

221 dominant limbs seem land with more control than dominant limbs in volleyball athletes. This would

222 suggest a greater level of joint control, through a decrease of the number of functional degrees of

223 freedom allowed by the neuromuscular system. It has also been reported that after performing

224 preventive training, the knee coordinative response would be expected to change. In task constraint

225 when the direction of jump landing is changed, the pattern of angle-angle and angle-velocity plots

226 in lateral jump landing should have a similarity to the forward direction. Soft-landing style, more

227 flexion of hip and knee joints, which has been suggested to reduce the risk of lower injury during

228 landing in various direction (Sinsurin et al., 2017; Sinsurin et al., 2013). Further work to investigate

229 the effect of soft-landing styles on knee coordinate may provide a greater understanding of the

230 effect of training techniques to reduce injury mechanisms.

231 The findings of this study are specific to volleyball athletes, application of these findings

232 to other sports should be made with caution. Further studies are required to explore the coronal and

233 transverse plane hip and knee kinematics, and other athletic groups should be included to determine

234 if the patterns of knee and hip coordination are similar. Further factors that should be considered

235 include, gender differences, athletes with ACL insufficiency, recovering from ankle injury and

236 athletes with patellofemoral pain syndrome. Multi-direction jump landing could also be utilised to

237 investigate the effectiveness of lower limb rehabilitation and risk of re-injury.

238

239

240 


\section{Conclusion}

The current study determined that direction of jump landing significantly influenced knee

243 flexion excursion and knee angular velocity during landing. In volleyball athletes, poor knee

244 coordination was observed in the early phase of lateral landing compared to forward and diagonal

245 directions. The non-dominant limb seems to land with better coordination than the dominant limb

246 during multi-direction jump landing. It may be possible to improve the control of the dominant

247 limb with training such as weight-bearing tasks to reduce risk of injury. Injury risk awareness

248 should be most concerned with lateral jump landing tasks in both limbs.

250 Acknowledgements

251 The authors would like to thank all the athletes for participation in this study.

\section{Disclosure statement}

$253 \quad$ No conflict of interest

\section{Funding}

255 This research project is supported by Mahidol University.

\section{References}

257 Bartlett, R. \& Bussey, M. (2012). Sports biomechanics: Reducing injury risk and improving sports 258 performance (pp. 229-243). New York: Routledge.

259 Boden, B.P., Dean, G.S., Feagin, J.A., \& Garrentt, W.E. (2000). Mechanism of anterior cruciate 260 ligament injury. Orthopedics, 23, 573-578.

261 Clack, J.E., \& Phillips, S.J. (1993). A longitudinal study of intralimb coordination in the first year 262 of independent walking: A dynamical system analysis. Child Development, 64, 1143-1157. 
263 Cronin, J.B., Bressel, E., \& Fkinn, L. (2008). Augmented feedback reduces ground reaction forces 264 in the landing phase of the volleyball spike jump. Journal of Sport Rehabilitation, 17, 148-159. 265 Devita, P., \& Skelly, W.A. (1992). Effect of landing stiffness on joint kinetics and energetics in the 266 lower extremity. Medicine and Science in Sports and Exercise, 24, 108-115.

267 Favre, J., Clancy, C., Dowling, A.V., \& Andriacchi, T.P. (2016). Modification of knee flexion 268 angle has patient-specific effects on anterior cruciate ligament injury risk factors during jump 269 landing. The American Journal of Sports Medicine, 44, 1540-1546.

270 Grieve, D.W. (1986). Gait patterns and the speed of walking. Biomedical Engineering, 3, 119-122.

271 Grood, E.S. \& Suntay, W.J. (1983). A joint coordinate system for the clinical description of three272 dimensional motion: application to the knee. Journal of Biomechanical Engineering, 105, 136$273 \quad 144$.

274 Heiderscheit, B.C. (2002). Variability of stride characteristics and joint coordination among 275 individuals with unilateral patellofemoral pain. Journal of Applied Biomechanics, 18, 110-121.

276 Hootman, J.M., Dick, R., \& Aqel, J. (2007). Epidemiology of collegiate injuries for 15 sports: 277 summary and recommendations for injury prevention initiatives. Journal of Athletic Training, $278 \quad 42,311-319$.

279 Kirkendall, D.T., \& Garrett, W.E. (2000). The anterior cruciate ligament enigma. Injury 280 mechanisms and prevention. Clinical Orthopaedics and Related Research, 372, 64-68.

281 Kurz, M.J., \& Stergiou, N. (2004). Applied dynamic system theory for the analysis of movement. 282 In N. Stergiou (Eds.), Innovative analyses of human movement (pp. 93-119). Illinois: Human $283 \quad$ Kinetics.

284 Laughlin, W.A., Weinhandl, J.T., Kernozek, T.W., Cobb, S.C., Keennan, K.G., \& O’Connor, K.M. 285 (2011). The effects of single-leg landing technique on ACL loading. Journal of Biomechanics, $286 \quad 44,1845-1851$. 
287 Onate, J.A., Guskiewicz, K.M., \& Sullivan, R.J. (2001). Augmented feedback reduces jump 288 landing forces. The Journal of Orthopaedic \& Sports Physical Therapy, 31, 511-7.

289 Richards, J. (2008). Biomechanics in clinical and research (pp. 64-65). Churchill Livingstone 290 Elsevier.

291 Robertson, G., Caldwell, G., Hamill, J., Kamen, G., \& Whittlesey, S. (2014). Research Methods in 292 Biomechanics (pp. 291-297). Illinois: Human kinetics.

293 Ross, S., Guskiewicz, K., Prentice, W., Schneider, R., \& Yu, B. (2004). Comparison of 294 biomechanical factors between the kicking and stance limbs. Journal of Sport Rehabilitation, $295 \quad 13,135-150$.

296 Scholz, J.P. (1990). Dynamic pattern theory: some implications for therapeutics. Physical Therapy, $297 \quad 70,827-843$.

298 Sinsurin, K., Srisangboriboon, S., \& Vachalathiti, R. (2017). Side-to-side differences in lower 299 extremity biomechanics during multi-directional jump landing in volleyball athletes. European $300 \quad$ Journal of Sport Sciences, 17, 699-709.

301 Sinsurin, K., Vachalathiti, R., Jalayondeja, W. \& Limroongreungrat, W. (2016). Knee muscular 302 control during jump landing in multidirections._Asian Journal of Sports Medicine, 7, e31248.

303 Sinsurin, K., Vachalathiti, R., Jalayondeja, W. \& Limroongreungrat, W. (2013). Different sagittal 304 angles and moments of lower extremity joints during single-leg jump landing among various 305 directions in basketball and volleyball athletes. Journal of Physical Therapy Science, 25, 11093061113.

307 Tamura, A., Akasaka, K., Otsudo, T., Schiozawa, J., Toda, Y. \& Yamada, K. (2017). Dynamic 308 knee valgus alignment influences impact attenuation in the lower extremity during the 309 deceleration phase of a single-leg landing. PLoS One, 12, e0179810. 
310 Tillman, M.D., Hass, C.J., Brunt, D., \& Bennett, G.R. (2004). Jumping and landing techniques in

311 elite women's volleyball. Journal of Sports Science \& Medicine, 3, 30-36.

312 van der Harst, J.J., Gokeler, A., \& Hof, A.L. (2007). Leg kinematics and kinetics in landing from 313 a single-leg hop for distance: A comparison between dominant and non-dominant leg. Clinical 314 Biomechanics, 22, 674-680.

315 William, G.N., Chmielewski, T., Rudolph, K., Buchanan, T.S., \& Snyder-Mackler, L. (2001).

316 Dynamic knee stability: current theory and implications for clinicians and scientists. The 317 Journal of Orthopaedic and Sports Physical Therapy, 31, 5466-5466.

318 Winter, D. A. (2005). Biomechanics and motor control of human movement (pp. 49-50). Waterloo: 319 John Wiley \& Sons.

320 Yu, B., Lin, C.F., \& Garrett, W.E. (2007). Mechanisms of non-contact ACL injuries. British $321 \quad$ Journal of Sports Medicine, 41, 147-151.

322 Zhang, S.N., Bates, B.T., \& Dufek, J.S. (2000). Contributions of lower extremity joints to energy 323 dissipation during landings. Medicine and Science in Sports and Exercise, 32, 812-819. 
324 Table 1. Mean \pm SD of the knee kinematics during jump landings in forward $\left(0^{\circ}\right), 30^{\circ}$ diagonal, $60^{\circ}$ diagonal, and lateral $\left(90^{\circ}\right)$

325 directions

\begin{tabular}{|c|c|c|c|c|c|c|c|c|c|c|c|}
\hline \multirow{2}{*}{ Dependent variables } & \multicolumn{4}{|c|}{ Non-dominant } & \multicolumn{4}{|c|}{ Dominant } & \multicolumn{3}{|c|}{ p-values } \\
\hline & $\mathbf{0}^{\circ}$ & $\mathbf{3 0}^{\circ}$ & $60^{\circ}$ & $90^{\circ}$ & $\mathbf{0}^{\circ}$ & $\mathbf{3 0}^{\circ}$ & $60^{\circ}$ & $90^{\circ}$ & Dominant & Direction & Interaction \\
\hline Angular velocity at initial & $235.3 \pm$ & $202.4 \pm$ & $160.5 \pm$ & $90.8 \pm$ & $238.0 \pm$ & $205.3 \pm$ & $183.2 \pm$ & & \multirow{2}{*}{0.649} & \multirow{2}{*}{$<0.001$} & \multirow{2}{*}{0.331} \\
\hline contact (degrees/sec) & $104.4^{\mathrm{a}, \mathrm{b}, \mathrm{c}}$ & $100.1^{\mathrm{b}, \mathrm{c}}$ & $108.3^{\mathrm{c}}$ & 68.6 & $94.6^{\mathrm{a}, \mathrm{b}, \mathrm{c}}$ & $108.2^{\mathrm{b}, \mathrm{c}}$ & $83.3^{\mathrm{c}}$ & 49.6 & & & \\
\hline Angular velocity at peak & $165.5 \pm$ & $161.3 \pm$ & $266.4 \pm$ & $366.9 \pm$ & $188.9 \pm$ & $174.5 \pm$ & $192.2 \pm$ & $412.8 \pm$ & \multirow{2}{*}{0.963} & \multirow{2}{*}{$<0.001$} & \multirow{2}{*}{0.212} \\
\hline VGRF (degrees/sec) & $181.6^{\mathrm{b}, \mathrm{c}}$ & $218.1^{\mathrm{b}, \mathrm{c}}$ & $221.0^{\mathrm{c}}$ & 243.7 & $220.6^{c}$ & $357.9^{c}$ & $269.1^{\mathrm{c}}$ & 324.8 & & & \\
\hline \multirow{2}{*}{ Flexion excursion (degrees) } & $38.9 \pm$ & $39.0 \pm$ & $38.9 \pm$ & $37.2 \pm$ & $40.3 \pm$ & $40.0 \pm$ & $40.0 \pm$ & $37.9 \pm$ & \multirow{2}{*}{0.398} & \multirow{2}{*}{0.016} & \multirow{2}{*}{0.926} \\
\hline & 6.2 & $5.4^{\mathrm{c}}$ & 5.7 & 4.9 & 6.3 & $5.4^{\mathrm{c}}$ & $4.9^{\mathrm{c}}$ & 4.8 & & & \\
\hline
\end{tabular}

$326{ }^{a}$ Statistically significant difference compared with $30^{\circ}$ diagonal direction $(<0.05),{ }^{\mathrm{b}}$ Statistically significant difference compared with

$32760^{\circ}$ diagonal direction $(<0.05),{ }^{\mathrm{c}}$ Statistically significant difference compared with lateral direction $(<0.05),{ }^{\mathrm{d}}$ Statistically significant

328 difference compared with dominant limb $(<0.05)$ 
332 Figure 1. Research setting in the laboratory (modified from Sinsurin et al., 2017). 70cm is

333 the distance from the starting point of jump-landing tests to the center of force plate. A,

334 lateral $\left(90^{\circ}\right)$ jump landing for the right lower limb; B, $60^{\circ}$ diagonal jump landing for the

335 right lower limb; C, $30^{\circ}$ diagonal jump landing for the right lower limb; $\mathrm{D}$, forward $\left(0^{\circ}\right)$

336 jump landing for the right and left lower limbs; E, $30^{\circ}$ diagonal jump landing for the left

337 lower limb; F, $60^{\circ}$ diagonal jump landing for the left lower limb; G, lateral (90) jump

338 landing for the left lower limb.

339

340 Figure 2. Knee flexion angle during landing of non-dominant knee (a) and dominant knee

341 (b). The $y$-axis is knee flexion angle (degrees). The $x$-axis is the time during landing phase

342 (300ms) which is normalised to $100 \%$ (\% normalised landing phase).

344 Figure 3. Knee angular velocity during landing of non-dominant knee (a) and dominant

345 knee (b). The $y$-axis is knee angular velocity (degrees/sec). The $\mathrm{x}$-axis is the time during

346 landing phase (300ms) which is normalised to $100 \%$ (\%normalised landing phase).

348 Figure 4. Hip-knee angle-angle plot during landing of non-dominant knee (a) and dominant

349 knee (b). The $\mathrm{y}$-axis is hip flexion angle (degrees). The $\mathrm{x}$-axis is knee flexion angle 350 (degrees).

351

352 Figure 5. Comparing pattern of knee velocity-angle plot between directions of non353 dominant knee (a) and dominant knee (b). The y-axis is knee angular velocity 354 (degrees/sec). The $\mathrm{x}$-axis is knee flexion angle (degrees). 
355 Figure 6. Comparing pattern of knee velocity-angle plot between non-dominant and 356 dominant limbs in various directions (a) at forward (0 degree) direction (b) at 30 degrees 357 diagonal (c) at 60 degrees diagonal (d) at lateral (90 degrees) direction. The y-axis is knee 358 angular velocity (degrees/sec). The $\mathrm{x}$-axis is knee flexion angle (degrees). 